\title{
MedienPädagogik
}

Zeitschrift für Theorie und Praxis der Medienbildung

Themenheft Nr. 42: Optimierung in der Medienpädagogik.

Forschungsperspektiven im Anschluss an den 27. Kongress der DGfE

Herausgegeben von Patrick Bettinger, Klaus Rummler und Karsten D. Wolf

\section{Zwischen Optimierung und ludischen Gegenstrategien}

\author{
Ästhetische Praktiken von Jugendlichen an der Social Media Schnittstelle \\ Viktoria Flasche und Anna Carnap
}

\begin{abstract}
Zusammenfassung
Wie lassen sich post-digitale Regime der Optimierung beforschen? Insbesondere Social Media Plattformen arbeiten in ihren diversen Anwendungsoptionen mit Konzepten des kollektiv und algorithmisch hergestellten Optimums der permanenten, potentiellen Verbesserung. Die Optimierungsappelle sind strukturell und dezentral in algorithmisch gesteuerte Prozesse eingelagert und erzeugen spezifische Affizierungsrelationen und Selbstbezüglichkeiten. Welche digital grammatisierten Positionierungspraktiken finden aktuell an der Schnittstelle zwischen digitaler Plattform und Jugendlichen statt? Mit dieser Fragestellung fokussieren wir in dem vorliegenden Beitrag exemplarisch das Medienhandeln von zwei 12-jährigen Mädchen mit und auf der Plattform TikTok, das im Rahmen der BMBF-geförderten DiKuJu-Studie (2016-2019) erhoben und analysiert wurde. Es konnte herausgearbeitet werden, wie an der Schnittstelle Freund*innenschaft gleichzeitig in Prozesse der Optimierung und ludischen Gegenstrategien eingebettet ist.
\end{abstract}

Between Optimization and Ludic Counter-Strategies. Aesthetic Practices of Young People at the Social Media Interface

\begin{abstract}
How can post-digital regimes of optimization be approached? In their diverse application options, social media platforms work with concepts of the collectively and algorithmically produced optimum of permanent improvement. Optimization demands are now structurally and decentrally embedded in algorithmically controlled processes and generate specific affliction relations and self-referentialities. What digital positioning practices are taking place at the interface digital platform-young people? With this question, this article focuses on the media actions of two 12-year-old girls on and with the TikTok platform, which was analyzed in the BMBF-funded DiKuJu study (2016-2019). It shows, how friendship is simultaneously embedded in processes of optimization and ludic counter-strategies at the interface.
\end{abstract} der Social Media Schnittstelle». MedienPädagogik 42, (Optimierung), 259-280. https://doi.org/10.21240/mpaed/42/2021.06.22.X. 


\section{Optimierung im Gefüge der Social Media}

Social Media Plattformen arbeiten durch ihre Anwendungsoptionen mit dem Konzept des Optimums und erzeugen so Affizierungsrelationen und Selbstbezüglichkeiten: Das am häufigsten aufgerufene Video, welches die Neugier weckt, das Bild mit den meisten Likes, das zur Referenz für die eigenen Bilder wird, oder der meist zitierte Artikel, der das Ranking in die Höhe treibt und so wissenschaftliche Diskurse mitsteuert.

"Schon immer sprachen Medien die Affizierbarkeit des Menschen an, versuchen sie zu mobilisieren und zu monetarisieren. Qualitativ neu im Web 2.0 jedoch ist, dass das Medium sich nun auch umgekehrt als empfänglich für oder gar interessiert an Affizierungen erweist, die von jeder einzelnen Nutzer ${ }^{\star}$ in ausgehen» (Breljak und Mühlhoff 2020, 17).

Optimierung funktioniert hier «freiwillig und lustvoll» und steht dennoch «prinzipiell im Dienst der technischen Apparate, ihrer Macht und ihrer Ausbeutungsmechanismen» (ebd., 18).

Der gleichsam drängende wie fortwährende Appell zur (Selbst-)Optimierung wurde als gouvernementale, vielleicht sogar epochemachende, moderne Praktik (Foucault 1977, 2000; Bröckling 2007) analysiert, d. h. Macht und Kontrolle entfalten sich nicht nur mittels äusserer Beeinflussung des Einzelnen (Sanktionen, Strafen, Folter), sondern vielmehr als innere Selbstführung. Mit (Selbst-)Erziehungstechniken und medizinisch fundierten Kontrollsystemen sind wir aufgefordert, stets das Beste zu leisten (Hoffarth 2012, 210f). Aus der Perspektive der/des Einzelnen bedeutet das, sich selbst als Projekt zu verstehen, als potentiell permanent optimierungsfähig, -bereit und -bedürftig. Hegemoniale Praktiken, d. h. solche der Ideologisierung, Differenzierung und Privilegierung durch Normalisierung (Marchart 2003; Forster 2005) sind mit gouvernementalen Praktiken weder identisch noch unverbunden oder widersprüchlich, sondern verschränkt (siehe auch Waldmann und Aktaş 2021). Zu den hegemonialen Praktiken zählen wir den affirmativen Umgang mit Verhältnissen der Über- und Unterordnung, die Abwertung von Individuen und Kollektiven, die nicht der neoliberalen Vorstellung vom weiss-männlichen und leistungsstarken Normalsubjekt genügen können oder wollen, d. h. Othering-Praktiken und ihre Gegenpraktiken. Auch der subversive Umgang mit hierarchisch organisierten Ordnungssystemen kann ex negativo dazu gezählt werden, insofern mit inm hegemoniale Verhältnisse bearbeitet werden oder diese als produktive Kontrastfolie dienen. Für die/den Einzelnen werden hegemoniale Praktiken in den (Un-)Möglichkeiten der selbstverständlichen und wertschätzenden Sichtbarwerdung relevant, in der (Un-)Möglichkeit, Bedeutungs- oder/und Leistungstragende zu sein, in dem Privileg, auf der Handlungsebene über Alternativen bzw. Auswahlmöglichkeiten zu verfügen oder ebensolche diskutieren und entwickeln zu können. Hegemonial-gouvernemental verschränkte 
Optimierungsfiguren vermitteln, verschieben und verändern sich gesellschaftlich, kulturell sowie intra- und intergenerational diskursiv bzw. medial, denn Medien - so Hall 1979 - sind «Konsensmanufakturen», in und mit innen artikuliert sich der common sense (ebd., 339 zitiert nach Marchart 2003, 13). Insofern sind Medien «nicht zu verstehen als Mittel der Übertragung von Botschaften, sondern als Institutionen der Erzeugung und Artikulation von konsensualer Bedeutung im Rahmen hegemonialer Auseinandersetzungen» (Marchart 2003, 13 mit Verweis auf Hall 1979, 339).

Mit der Gegenwartsdiagnose des «(post-)digitalen Zustands» und der Annahme einer «post-digitalen Kultur» (Berry 2014; Cramer 2014; Jörissen 2016; Stalder 2016) geht unsere Untersuchung von einer tiefgreifenden Mediatisierung der Alltagswelt aus (Hepp und Hasebrink 2013) und betont, dass «Strukturen, die sich aus Digitalisierungsdynamiken ergeben - wie beispielsweise die Externalisierung von Gedächtnispraktiken, Sozialität als netzwerkförmige Aufmerksamkeitsökonomie, Kreativitätsimperative oder hyperindividualisierte Informations- und Kommunikationsstile - begonnen haben, auch nicht digitale, also nicht unmittelbar technische Lebensvollzüge zu strukturieren bzw. zu restrukturieren» (Jörissen, Schroeder, und Carnap 2020, 61). Der postdigitale Zustand wird unter anderem daran erkenntlich, dass jetzt das «Nicht-Digitale» einen Namen braucht (ebd.), beispielsweise die ästhetische Praktik der Analogfotografie oder das Lehr-Lernformat des Präsenzunterrichts. Ein nicht-digitaler Vorgang muss explizit gemacht werden, er ist nicht mehr selbstverständlich. Das Digitale dagegen ist - insbesondere für Jugendliche - «kein Gegenstand der Thematisierung, sondern konstitutiver, unhintergehbarer Hintergrund für das Erscheinen von Lebenswelt» (ebd., 65), ein permanentes «Hintergrundrauschen» (ebd.), das auch als ein ins «real life gestülpte[r] Cyberspace» (Meyer 2012, 30) beschrieben worden ist.

Bevor das Erkenntnisinteresse an der postdigitalen Differenz in Richtung Forschungsfrage und -methodik zugespitzt wird (Wie lassen sich postdigitale Konsensmanufakturen beforschen? Wie können bewährte Analyseschritte der rekonstruktiven Sozialforschung gegenstandsangemessen weiterentwickelt werden?), ist für den vorliegenden Zusammenhang bemerkenswert, dass in der "Kultur des Digitalen» (Stalder 2016) gerade die Sichtbarkeit eine zentrale Rolle spielt. Wenn wir den subjektivierenden Optimierungsbogen von gouvernemental/hegemonial zu medial/digital hin zu postdigital/sozial spannen, gilt es, sich mit dem Digitalen (im Postdigitalen) noch etwas weiter zu beschäftigen.

Mit Bezug auf Andreas Reckwitz (2008) macht Hannelore Bublitz bereits 2010 deutlich, dass die Subjekte des digitalen Zeitalters sich «nicht primär als Innenwelten, sondern als visuelle performances (re-)präsentieren» und so gleichsam zu "Objekte[n] des being-looked-at-ness» werden (ebd., 104). Neben Zahlen und Daten ist die «Verschränkung von Visualität, Macht und Reflexivität [...] ein zentrales Prinzip digitalisierter Kultur» (Jörissen 2016, 12). Gerade Social Media Plattformen machen 
es notwendig, sich als ein Sichtbares zu gestalten, um im medialen Raum anerkennbar zu sein, d. h. intelligibel agieren zu können (Schaffer 2008, 151) ${ }^{1}$. Sichtbar-zu-sein fassen wir als einen hegemonialen Anspruch der untersuchten Social Media Plattformen auf, wohingegen sich die Praxis des Sichtbarwerdens auf Ebene ästhetischer Artikulationen nach gouvernementalen Strukturmerkmalen organisiert. Es existieren (meist) keine expliziten Vorgaben oder Normen, an denen sich die visuellen Artikulationen orientieren könnten, vielmehr regulieren marktförmige und kompetitiv-kreative Sichtbarkeitsordnungen die «Sphäre des Erscheinens» (Butler 2010, 9)².

Wie lassen sich post-digitale Regime der Optimierung beforschen? Wenngleich Social Media Plattformen massenhaft genutzt werden und die Anzahl der verschiedenen Plattformen bzw. der Anbieter/Unternehmen überschaubar ist, unterscheiden sie sich grundlegend von den massenmedialen Konsensmanufakturen Fernsehen, Kino und Druckerzeugnissen. Die Konsensmanufakturen der heutigen Zeit sind nicht mehr ausschliesslich analog konzipiert, sondern digital-persistent, (global) vernetzt, reaktiv und medien-konvergent (Boyd 2014, 11). Es gibt keinen an eine Masse gerichteten, zentralen, öffentlichen, one-way-kommunizierten Content mehr, der auf seine spezifischen Optimierungsappelle, Repräsentationslogiken und Blickregime hin analysiert werden könnte - wie es beispielsweise den Kultur- und Medienforschenden Hall (1989), Silverman (1997), Poster (2008) und McRobbie (2010) noch möglich war. Denn: Social Media Plattformen agieren auf die Userinnen- und Useraktivität hin und mit ihr. Ihr Organisationsprinzip ist nicht linear oder hierarchisch, sondern grundsätzlich, auf allen Ebenen der Organisation, netzwerklogisch strukturiert.

Nassehi (2019) geht davon aus, dass sich Herrschaft in der digitalen Gesellschaft als «Schnittstellenmanagement» entfaltet (ebd., 238). Optimierungsappelle sind nun strukturell und dezentral in algorithmisch gesteuerte Prozesse eingelagert, z. B. über die gezielte Anzeige von Inhalten, die besonders viele Likes aufweisen. Die strukturelle (Herrschafts-)Dimension als zentrales Moment digital-mediatisierter Diskursbewegungen betont auch Traue (2013) mit Stieglers Begriff der «Grammatisierung» (ebd., 9 mit Verweis auf Stiegler 2009 und Lury 1997). Über die Grammatisierung treiben digitale Medien eine «Formalisierung und Diskretisierung der Handlungsformen» voran, die das Handeln gleichsam «organisiert» und «orientiert» und dabei «nicht nur Wahrnehmungsweisen, Erinnerungsweisen und Entwürfe [stiftet], sondern auch Positionierungen im Diskurs» (ebd.).

Welche digital grammatisierten Optimierungs- und Positionierungspraktiken finden aktuell an der Schnittstelle digitale Plattform-Jugendliche statt? In dem vorliegenden Beitrag analysieren wir exemplarisch das Medienhandeln von zwei

1 Der Verbildlichung ist in sozialen Medien nahezu unterhintergehbar. Nutzer*innen, die nicht in Erscheinung treten wollen, werden i.d.R. von einer pattformspezifischen Silhouette repräsentiert, die einerseits auch ein Bild ist und zudem Auskunft über die Nutzer*in gibt (das ist jemand, die* der kein Bild zur Selbstdarstellung wählt).

2 Selbst im Fall der direkten Zensur kann nur im Nachhinein auf wahrscheinliche, intern vorhandene Veröffentlichungsregeln geschlossen werden. 
12-jährigen Mädchen mit und auf der Plattform TikTok, das im Rahmen der BMBFgeförderten DiKuJu-Studie (2016-2019) erhoben und interpretiert wurde. Wir verstehen die digital-visuellen Artikulationen grundsätzlich als Erzeugnisse künstlerisch-kreativer Hybridsubjekte, die aus einer techno-sozialen Herstellungspraxis hervorgehen. Diese Perspektive macht es notwendig, in die Fallanalyse, neben den medialen Artefakten, auch eine Strukturanalytik der Plattformen miteinzubeziehen. Im Anschluss diskutieren wir die Analyseergebnisse hinsichtlich der gouvernementalen und hegemonialen Optimierungsfiguren, die in dem plattformspezifischen Feld des Erscheinens (re-)produziert und bearbeitet wurden und um das Thema der Freund ${ }^{\star}$ innenschaft kreisen. Abschliessend fragen wir, welche Funktionen diese Optimierungsfiguren im Kontext der Regime des Normalen und der Prekarisierung einnehmen (siehe auch Waldmann und Aktaş 2021).

\section{Post-Digitale Jugendwelten aus transaktionaler Perspektive}

Das Projekt «Postdigitale Kulturelle Jugendwelten» untersuchte im Erhebungszeitraum von 2016 bis 2019, wie sich der digitale Wandel speziell auf die künstlerischkreative Praxis junger Menschen ausgewirkt hat ${ }^{3}$. Das Mixed-Methods Forschungsdesign umfasste neben einer quantitativ-repräsentativen Interviewstudie unterschiedliche qualitative Erhebungsszenarien und Analyseeinstellungen ${ }^{4}$.

Die beforschten Jugendlichen waren im Untersuchungszeitraum von 2016 bis 2019 12-24 Jahre alt und können somit im Sinne einer Generationeneinteilung auf der Grenze zwischen den Generationen $Y$ und Z verortet werden (Albert, Hurrelmann, und Quenzel 2019). Gerade für Letztere wurden die intelligenten Technologien und sozialen Medien als allgegenwärtige Merkmale des täglichen Lebens herausgearbeitet (ebd., 40).

(Post-)digitale ästhetische Praktiken sind gekennzeichnet durch digitales Handlungswissen, das die alltäglichen, selbstverständlichen Medienpraktiken implizit anleitet, den Beforschten aber nicht (unbedingt) reflexiv zur Verfügung steht. Es wurden videogestützte, teilnehmende Beobachtungen und transaktionale (Kleingruppen-)

3 Das Verbundprojekt «Postdigitale kulturelle Jugendwelten - Entwicklung neuer Methodeninstrumente zur Weiterentwicklung der Forschung zur Kulturellen Bildung in der digitalen und postdigitalen Welt» wurde unter der Leitung von Benjamin Jörissen an der Friedrich-Alexander-Universität Erlangen-Nürnberg und Susanne Keuchel am Institut für Bildung und Kultur und der Akademie der Kulturellen Bildung Remscheid durchgeführt und durch das BMBF gefördert (Jörissen, Schroeder, und Carnap 2020).

4 Die erste Phase des qualitativen Teilprojekts konzentrierte sich auf die Entwicklung eines Überblicks über die (post-)digitale ästhetische Praxis junger Menschen, wobei vor allem deskriptive methodische Ansätze verwendet wurden, unter anderem Expert*inneninterviews mit Fachleuten aus dem Bereich der kulturellen Bildung und Gruppendiskussionen mit Jugendlichen, die im Umfeld kulturbezogener Institutionen, wie zum Beispiel Schulen mit kulturellem Schwerpunkt oder Jugendmedienzentren, erhoben wurden. Diese Ergebnisse dienten unter anderem der Itementwicklung der Umfrage-Studie des quantitativen Teilprojektes. Die zweite qualitative Phase umfasste die Durchführung und rekonstruktive Auswertung eines Barcamps sowie transaktionale Interviews mit Jugendlichen und die teilnehmende Beobachtung eines Festivals. In dieser Phase wurde ein sinnverstehender sowie strukturanalytischer Ansatz mit dem Schwerpunkt auf digitale Jugendkulturen verfolgt (Jörissen, Schroeder, und Carnap 2020). 
Interviews geführt (Nohl 2011; Engel und Jörissen 2018). Eine transaktionale Perspektive wurde bisher im Bereich der erziehungswissenschaftlichen Forschung vor allem zur Einbeziehung von Räumen und materiellen Dingwelten genutzt (Nohl 2017). Die transaktionale Forschungsmethodik eröffnet durch den Wechsel der Analyseeinstellung auf das konstitutiv wechselseitige, relationale Verhältnis von physischen, materiellen sowie räumlichen Akteurinnen und Akteuren die Möglichkeit, «die Genese dieser Entitäten aus transaktionalen Praktiken zu rekonstruieren» (ebd., 1). Im Sinne der Akteurs-Netzwerk-Theorie (Latour, 1998) wird das Ineinandergreifen von menschlichen sowie nicht-menschlichen Akteurinnen und Akteuren im Vollzug der Handlung und nicht a priori subjektzentriert hierarchisiert erforscht. Gerade dem Spezifischen ihrer gegenseitigen Verhältnishaftigkeit gilt das empirische Erkenntnisinteresse. Aus dieser Forschungsperspektive heraus ist die rekonstruktive Artefaktanalyse, die auf die Eigensinnigkeit des Gegenstandes abhebt, ein zentraler Arbeitsschritt (Nohl 2017, 115).

Mit dieser Perspektive reagieren wir auf die methodische Herausforderung der Postdigitalität, die sich wie beschrieben u. a. durch Reaktivität und Medienkonvergenz auszeichnet. Für die transaktionale Rekonstruktion (post-)digialer Praktiken haben wir die vorgesehene Artefaktanalyse in eine Analyse der von den Jugendlichen an der Schnittstelle produzierten Inhalte und der relevanten Social-Media-Plattformen übersetzt. Die Plattformanalyse orientiert sich an der Social-Media-Analyse von VanDijcks und Poell (2013), die Geschäftsmodell, Governance, (Meta-)Daten-Algorithmen, Protokolle, Schnittstellen und Voreinstellungen berücksichtigt. Insbesondere die Schnittstelle ist für unsere Analyse entscheidend: Die Schnittstelle ist das Produkt aller informationellen und strukturellen Merkmale der jeweiligen Plattform und stellt im Sinne der o. g. Grammatisierung eine Vorformalisierung von Praktiken der Userinnen und User dar. Denn es sind nur die Anwendungen möglich, die durch die Einstellungen der Plattform bereitgestellt werden. Auch wenn sich algorithmische Entscheidungsprozesse in Bezug auf die Nutzendenaktivität reaktiv umstrukturieren können, sind alle Aktivitäten nur in den Kategorien möglich, die im Sinne der Plattformlogik geschaffen wurden (Poell 2020, 653). Die an der Schnittstelle vorgesehenen Interaktionsmöglichkeiten positionieren den/die Nutzende auf bestimmte Weise und evozieren so - durchaus produktive - Subjektivierungsprozesse.

«Der den Dingen immanente Entwurfscharakter, das von ihnen verkörperte, strukturimmanente Wissen betrifft nicht nur ihre «Gebrauchsmöglichkeiten), sondern, auf einer theoretisch tieferliegenden Ebene, ihre Relationierungspotenziale in Hinblick auf andere (dingliche oder menschliche) Akteure. Im Rahmen von Alltagspraktiken stellen Dinge und Dingumwelten Subjektivationsangebote dar, also insbesondere Angebote, auf bestimmte Weise zu Nutzer-Subjekten (Konsumenten, Rezipienten, Prosumenten etc.) zu werden» (Jörissen 2014, 218). 
Wenn Social Media Plattformen zum Hinzufügen, Teilen bzw. Posten eines (Bild-) Beitrags auffordern - indem sie diese Funktion anbieten (vgl. Abschnitt 3.2) - bringen sie die Anwendenden bspw. in die Position, potentiell einen (Bild-)Beitrag teilen zu wollen. Traue (2013) zufolge ist es gerade der (Bild-)Autorisierungsmoment, der die Initiative der Anwendenden lokalisiert und das Mitwirken der Plattform invisibilisiert, der neue, also hybride Subjektpositionen schafft (ebd., 9f.).

«Die Medien und Kulturen des (Selbermachens), der (mass self-communication> ermutigen Autorisierung und Selbstautorisierungen, also neue Sprecherund Subjektpositionen» (ebd., gf mit Verweis auf Castells 2008, 58ff.).

So bedingen etwa unterschiedliche "Upload-Dramaturgien» (Schreiber 2020) unterschiedliche Sichtbarkeitslogiken und Öffentlichkeiten, die - trotz oder gerade wegen ihrer begrenzten und fest vorinstallierten (Re-)Aktionsmöglichkeiten - ein spezifisches Know-How erfordern. Z. B. haben

«Beiträge in halböffentlichen Plattformen [...] das Potential von vielen gesehen zu werden, aber um zu zeigen, dass etwas gesehen wurde, muss auf Like oder Herz geklickt werden - sonst ist es für das Gegenüber nicht ersichtlich. Diese Feinheiten und Politiken des Sehens und (Nicht-)Reagierens können Schmiermittel und Zündstoff für zwischenmenschliche Beziehungen sein» (Schreiber 2020, 99).

Hier zeigt sich, wie jede Schnittstelle ein postdigital-soziales Können verlangt, das es zu beherrschen gilt, um mitspielen zu können. Oder, subjektivationstheoretisch gewendet, sie verlangt Unterwerfung (unter die plattformspezifischen Bedingungen der Sichtbarkeit und Berechenbarkeit), im Gegenzug bietet sie eine (ebenfalls plattformspezifische) Handlungsfähigkeit an.

\section{Das Hybridsubjekt Lara und Lara | TikTok}

Innerhalb des Erhebungssettings des DiKuJu Projektes zielten die transaktionalen Interviews darauf, eine grössere Klarheit über dezidiert (post-)digitalisierte Kreativpraktiken innerhalb jugendlicher Lebenswelten zu erhalten. Gerade hier zeigte sich, wie materielle, ästhetisch-kreative und künstlerische Praktiken sich auf unterschiedliche Weise mit digitalen Technologien und Anwendungen verschränken. Im Rahmen unserer Analysen kommen wir dem künstlerischen Hybridsubjekt - bestehend aus Interviewee(s), Artefakten, Media Plattform(en) - auf die Spur. ${ }^{5}$

5 Die Artefakte stellen visuelle, d. h. Bild- oder Videodaten dar. Sie wurden den Forschenden im Interview etwa auf die Nachfrage: «Magst du mir mal so ein Bild/Video zeigen?» oder «Darf ich mal sehen?» gezeigt. Das Zeigen (Sehen-Lassen) deuten wir als Autorisierung einerseits und Relevantsetzung andererseits (Przyborski 2018). 
Lara und Lara sind zwei 12-jährige Interview-Teilnehmerinnen, befreundete Nachbarinnen, die den gleichen Namen tragen (pseudonymisiert als Lara und Lara), die uns ihre TikTok Videos zeigen. Vor dem Hintergrund der repräsentativen JIM-Studie 2019 ist ihre Medienpraxis - die Favorisierung und häufige Nutzung der Plattform TikTok - (micro)generational- und geschlechtstypisch (Feierabend u. a. 2020) ${ }^{6}$. Die TikTok Videos von Lara und Lara bilden den Ausgangspunkt für die hier vorgestellte Analyse, wobei die Relevantsetzung - das Zeigen genau dieser Videos in der Interviewsituation - nicht einseitig bei den Interviewten festgemacht werden kann. Die ‘persönlich) algorithmisierte Plattform-Startseite der Beforschten priorisiert je bestimmte Bilder/Videos, die der Nutzenden oben im Feed angezeigt werden und so - einer Formulierung von Sigrid Schade und Silke Wenk (2011) folgend - zum Anschauen vor-gesehen sind. Das heisst, die Relevantsetzung von Bildern und Videos, die die Interviewten über Social Media Apps herstellen und teilen und im Interview thematisieren, kann bereits als eine sozio-technische Hybridentscheidung von Interviewees und Plattform verstanden werden. Der Zeige-Praxis liegt eine Relevanzordnung zugrunde, die auf Seiten der Plattform zunächst als algorithmisch organisiert gefasst werden kann und auf Seiten der Nutzenden als praxeologisch und diskursiv strukturiert. Der Algorithmus arbeitet mit den berechenbaren Daten und Präferenzen der Nutzenden, die er je nach Programmierung der Plattformbetreibenden für seine ökonomisch motivierten Zwecke operationalisiert (Jörissen 2020, Carnap und Flasche 2020, zur berechnenden und produktiven Eigenlogik von Algorithmen siehe auch Roberge und Seyfert 2017).

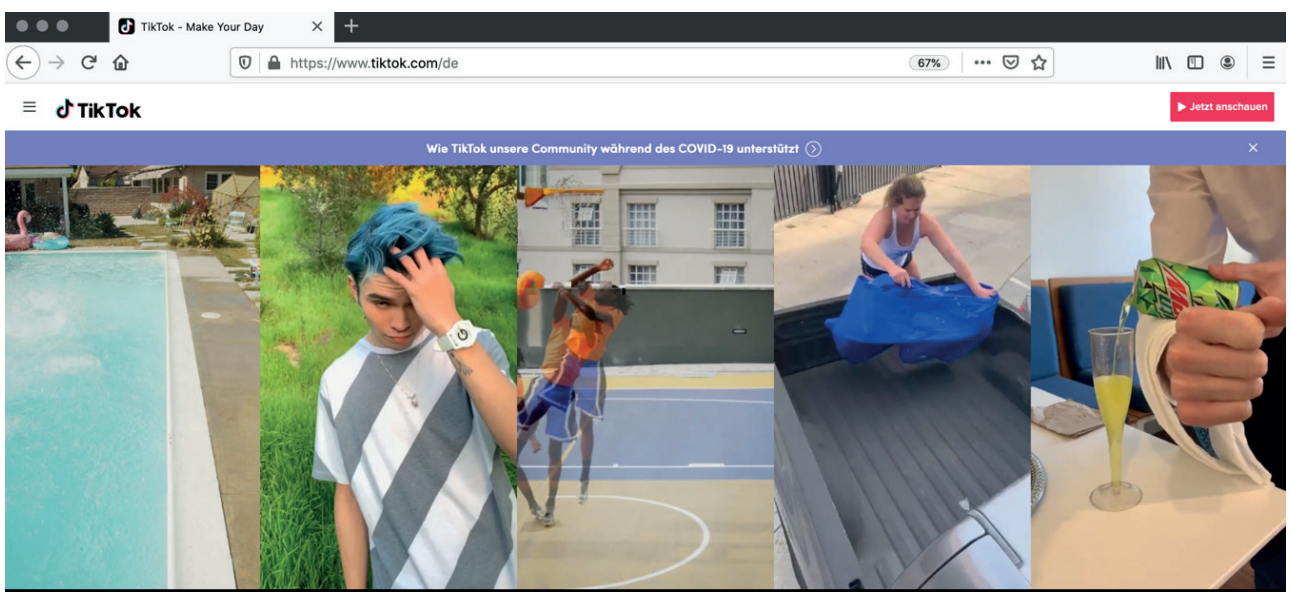

Abb. 1.: Screenshot der TikTok-Startseite abgerufen in der Browserversion im Herbst 2020 https://www.tiktok.com/de-DE.

6 Die JIM Studie unterscheidet hierbei dichotomal zwischen männlich und weiblich. 


\subsection{TikTok als machtvolle Schnittstelle}

Im Folgenden werden die charakteristischen Merkmale und zentralen Ergebnisse unserer Analyse der Social Media Plattform TikTok zusammengefasst. Dabei liegt der Fokus auf jenen Aspekten, die für die transaktionale Auswertung relevant geworden sind. Aufgrund der reaktiven und dynamischen Konzeption TikToks muss diese Analyse auf eine Momentaufnahme zum Zeitpunkt der Erhebung im Herbst 2018 beschränkt bleiben? ${ }^{7}$.

Die Plattform TikTok bietet eine Auswahl an Tonspuren, wie zum Beispiel Minidialoge oder Musiksequenzen, die von Nutzenden mit einem - meist selbst aufgenommenen/gefilmten - Video verknüpft werden. TikTok ist aktuell die am schnellsten wachsende Social Media Plattform weltweit (Firsching 2020). Charakteristisch für TikTok ist der wilde, für jeweilige Nutzende scheinbar ungeordnete, geloopte Video-Feed (siehe Abb. 1) mit seiner individuell-algorithmisch gesteuerten Anzeige, der auf ein Flow-Erleben zielt (Porombka 2016) und eine Übersteigerung von Anrufungen und Affizierungsrelationen evoziert (Carnap und Flasche 2020). Der Betreibernde versucht mittels zensorischer Prozesse «kontroverse» Inhalte z. B. mit Bezug zu politischen Konflikten zu löschen und gezielt nur - laut investigativer Recherche von netzpolitik.org 2019 - unterhaltsame «Gute Laune»-Videos zu zeigen (Reuter und Köver 2019). Die internen Richtlinien sind nicht öffentlich, sondern lassen sich nur implizit aus dem Löschungs- bzw. Rankingprozess ableiten. Die Journalistinnen und Journalisten haben nachweisen können, dass im Zeitraum ihrer Recherche Beiträge, die Konflikte der autonomen chinesischen Republiken (Xinjang, Tibet, Hongkong) erwähnen oder körperliche Behinderungen zeigen, gelöscht wurden oder ein nachteiliges Ranking erhalten haben (ebd.).

Social Media Plattformen wie TikTok arbeiten i. d. R. auf der Grundlage desselben Geschäftsmodells, das darauf zielt, die Anzahl der Personen, die auf der Plattform angemeldet sind und sich dort engagieren, zu erhöhen, um sie mit personalisierter Werbung gezielt ansprechen zu können (Poell 2020). Ferner bietet TikTok Geschäftskunden einen gezielten Verkauf von über die Plattform gesammelten Daten, hauptsächlich für Marktforschungszwecke (TikTok 2021). So entstehen plattformspezifische reaktive Konvergenzräume, in denen unterschiedliche Medienformen wie Bilder, Texte oder Bewegtbilder zusammenlaufen. Die algorithmisch gesteuerten, virtuellen Gestaltungsspielräume nehmen Aktivitäten (Handlungsmuster) ihrer Nutzenden auf,

7 Zum Zeitpunkt der Verfassung dieses Beitrags Mitte des Jahres 2020 ist die Zahl der Nutzenden und Aktivität stark angestiegen. Aufgrund steigender Bekanntheit des Netzwerkes ist sowohl die Zielgruppe breiter geworden, als auch die plattformtypische Verhandlung politischer Diskurse in den kritischen Fokus der Öffentlichkeit geraten. Die Plattform hat unter anderem durch neue Medienformate, wie längere Videos und eine Liberalisierung geduldeter politischer Inhalte, auf diese Kritik reagiert (Medina Serrano, Papakyriakopoulos, und Hegelich 2020). Aktuell zeichnet sich eine Entwicklung in national-diversifizierte Netzwerkstrukturen ab, um auf die jeweiligen nationalen Kontrollmechanismen flexibel reagieren können. Das mediale Alleinstellungsmerkmal wird zudem auch von anderen Plattformen, wie zum Beispiel den 〈reels` bei Instagram, adaptiert. 
verstärken sie und können reaktiv darauf ausgerichtet auch neue Funktionen entwickeln. Das heisst, Social-Media-Plattformen sind nicht nur ökonomisch, sondern konstitutiv von den Aktivitäten ihrer Userinnen und User abhängig.

Nassehi bescheinigt unter Verweis auf die Erkenntnisse einer informatorischen Grundlagenforschung (LeCun, Bengio, und Hinton 2015 zitiert nach Nassehi 2019, 234), dass die derzeit in einigen Social Media eingesetzten Algorithmen in der Lage sind, eine «selektive intrinsische Aktivität» zu entwickeln (ebd., 238). Auf der Grundlage der Analyse von Aktivitäten der Nutzenden sind die Algorithmen in der Lage, $a b$ duktiv eigene Schlussfolgerungen zu ziehen, die über das hinausgehen, was menschliche Akteurinnen und Akteure programmatisch entworfen haben (ebd., 241). So verstärken und erzeugen die TikTok Algorithmen von ihnen wiedererkennbare Muster, wie zum Beispiel visuelle Trends, die Nutzung von bestimmten Hashtags oder sogenannte (Challenges), auf Basis der bereits eingespeisten Aktivitäten der Userinnen und User. Wenn die Apps in der Lage sind, mit reaktiven algorithmischen Entscheidungsprozessen die Aktivität der Nutzenden zu erhöhen, steht der Plattform mehr Zeit zur Verfügung, personenbezogene Werbung gezielt zu platzieren. Gleichzeitig geht die Werbung auf TikTok vollständig im Fluss der Videos auf, da zum Beispiel Influencerinnen und Influencer das beworbene Produkt in ihre Videos integrieren ${ }^{8}$.

\subsection{Hybride Freund *innenschaften im Interview}

Lara und Lara orientieren sich bei ihren digitalen Aktivitäten an einer gleichgeschlechtlichen Peergroup aus Freundinnen und den von ihnen sogenannten «Internet-Best-Friends». Die Gruppe ist uneindeutig begrenzt und gerankt. Aus ihren Erzählungen geht hervor, dass jede Gleichaltrige und Gleichgeschlechtliche prinzipiell zugehörig ist. So werden Videos von eigentlich fremden Mädchen im Interview genauso gezeigt, wie selbst produzierte Videos. Das Motiv des «gegenseitigen Folgens» als Zugehörigkeits-Anzeige eines diffus offenen Freundinnen-Konzeptes ist Teil der Eröffnungssequenz im Interview und taucht im weiteren Verlauf wiederholt auf.

Interviewauszug Lara und Lara 00.01.42-00.02.51

Interviewerin: Was habt ihr gerade eben gemacht? als ihr (da am Computer wart)

L1: $\quad$ Wir haben Minecraft gespielt.

L2: $\quad$ Mein Computer muss updaten. Deswegen.

Interviewerin: Spielt ihr das zusammen?

L2: $\quad$ Ja. (.) Auf dem Server (.)

Interviewerin: Und spielt ihr das viel?

8 Anders als bei Youtube oder Instagram hat sich auf TikTok (noch) keine Kennzeichnungskonvention geförderter Inhalte herausgebildet. 
L1: $\quad$ Äh, @die letzten zwei Tage haben wir die ganze Zeit zusammen gespielt@ ((lachend gesprochen))

L2: $\quad$ Ja halt am Abend mit ihren Internetbestenfreunden. Oder mit ihren Internetfreunden. ${ }^{\circ}$ Nicht unbedingt besten Freunden ${ }^{\circ}$ ((leise gesprochen))

Interviewerin: Was sind denn Internetfreunde?

L2: $\quad$ Also die lernste über das Internet kennen und ihr versteht euch gut und dann seid ihr sozusagen wie jetzt im echten Leben beste Freunde dann seid ihr (übers) Internet beste Freunde.

Interviewerin: Mhm. (.) Und mit denen spielst du zusammen.

L1 und L2: J Ja. // Ja, ich auch. (1)

Interviewerin: Verabredet ihr euch dann?

L1 und L2: J Jain. // @Jain@ ((lachend gesprochen)) [...]

L1: $\quad$ Auf musicaly also TikTok, da sind wir schon (1) da sind wir schon.

L2: $\quad$ Da folgen wir uns gegenseitig.

Zentral für die Nutzung der Plattform TikTok ist für Lara und Lara das Motiv der Zugehörigkeit zu einer Gruppe gleichaltriger Mädchen bzw. zu einer besten Freundin. Das Prinzip der Zugehörigkeit erscheint nicht nur im Interview als handlungsleitend, sondern auch in den Videos, die sie im Interview zeigen (vgl. Abschnitt 3.4).

Die im Interview thematisierte Freund ${ }^{*}$ innenschaft definiert sich über Kreativität, Individualität und normative (geschlossene) Urteile. In einer Erzählung grenzen sie sich etwa von einem Mädchen ab, welches sie «einfach nachgemacht» hat. Dieses Mädchen hat auf TikTok «unseren Namen genommen.» Und das habe sie (Lara) «halt bisschen genervt» (00.14.05). Sie melden das Mädchen zwar nicht bei dem Plattformbetreibenden, weil sie ihr Handeln als «auch nicht sooo tragisch» einschätzen (ebd.), freunden sich aber auch nicht mit ihr an. Auch innerhalb ihrer ZweierFreund*innenschaft stecken Lara und Lara Domänen ab, was auf den identitätsstiftenden Charakter der Kreativpraxen verweist:

Interviewauszug Lara und Lara 00.06.59

L2: $\quad$ Ja. Also ich mach dis mit minecraft und sie macht es mit sich selber.

L1: @@Normal halt@o((lachend und leise gesprochen))

Sie sind für den «Erfolg` ihrer Videos sensibilisiert:

L2: $\quad$ «Es gibt ja zwei Minecraft-Youtuber und die haben wir imitiert. Und (.) und das war sehr erfolgreich. bei mir halt» (00.10.58). 
Dieses sich voneinander abgrenzende und tendenziell kompetitive Moment ist doppelt in der Plattform angelegt, da Rankings und Likes in Form von Herzchen sowohl direkt über jedem Video eingeblendet werden, als auch zentral für die nicht einsehbare Reihenfolge der Anzeige im Feed sind. Indem die algorithmischen Entscheidungsprozesse die Verbindung von einzelnen Userinnen und User intransparent prä-formalisiert, wird Freund*innenschaft grammatisiert und damit ungreifbarer. In Abgrenzung zu schon länger bestehenden Plattformen wie Facebook, auf denen das Freund ${ }^{*}$ innenschaftsmotiv ein zentrales und explizites Organisationsprinzip ist, wird die User*innen-Sozialität bei TikTok (und allen seit ca. 2010 etablierten und bei Jugendlichen beliebten Social Media Plattformen) unter dem Motiv des Folgens organisiert: Die TikTok User*in hat Follower und/oder ist selbst Follower. Lara und Lara und ihre Internet(-best)-friends sind Freundinnen, die sich gegenseitig folgen, sich aneinander orientieren, aber nicht kopieren. Nur wer es schafft, sich immer wieder neu im Dickicht aus Bild-, Video- und Audio-Zitaten originell und distinktiv zu verorten, kann Teil ihrer Gemeinschaft werden.

Zusammengefasst zeigt sich im Interview, wie sich Lara und Lara in ihrer Social Media-Praxis an kompetitiven Abgrenzungsmotiven orientieren und gleichzeitig an Motiven der Zugehörigkeit. Die divergenten Orientierungen ihrer hybriden Freund ${ }^{\star}$ innenschaft tritt in den Videos der Interviewten noch deutlicher hervor.

\subsection{Hybride Freund *innenschaft in den Videos}

Die uns von Lara und Lara als Teil des Interviews gezeigten Videos können zwei formal-heuristischen Kategorien zugeordnet werden: Zum einen die Selfie-Videos, die im Zentrum der Darstellung eine Person zeigen. Zum anderen die Mashup-Videos, die keinem eindeutigen Genre oder Modus zugeordnet werden können, sondern zwischen Musik- bzw. Playbackvideo und Animation changieren.
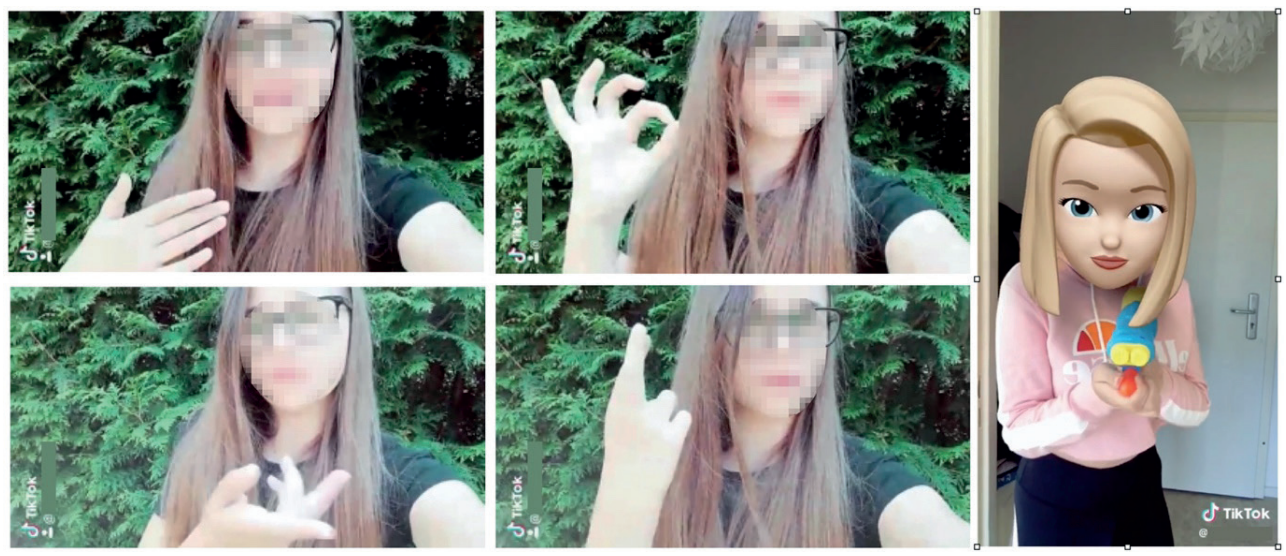

Abb. 2.: Screenshots aus den Selfie-Videos @Anna Carnap u. Viktoria Flasche. 
Exemplarisch für das Selfie-Video sind das «Ich hatte Glück mit meiner besten Freundin» und das «Baby can't you see i'm calling»-Video. In Ersterem ist ein von einer erwachsenen Stimme gesprochener Monolog Rahmen-gebend; eine Tonspur, die von TikTok zur Verfügung gestellt und von Lara gewählt wurde: «Ich hatte Glück mit meiner besten Freundin. Meine beste Freundin ist witzig. Intelligent. Kreativ. Wunderschön.» Die Tonspur fungiert als Maske (Carnap und Flasche 2020 mit Bezug auf Hoffarth 2012 und McRobbie 2010), denn sie bietet Lara die Möglichkeit, in die stärker vergeschlechtlichte Matrix der Erwachsenenwelt performativ einzutauchen und sichtbar zu werden, bzw. das Feld des Erscheinens zu betreten. Lara tut dies, indem sie sich auf ihre perfekte beste Freundin bezieht, mit der sie Glück hatte, sowie mit einer gekonnten Performance: Ruhig folgt sie dem Playback der erwachsenen Frauenstimme, souverän reiht sie eine Pose an die nächste.

Das zweite Video zeigt eine TikTok-Freundin von Lara und Lara als personalisierten Avatar, der zu dem Lied «Baby can't you see I'm calling» von Britney Spears tanzt. In den Tanz integriert sind unter anderem Kampfsport-Elemente und eine Supersoaker-Wasserpistole. Hier wird das Feld des Erscheinens mithilfe einer postdigital-perfektionierten Körper-Figur betreten, deren Anruf - so der Liedtext - nicht entgegengenommen wird. Sie artikuliert ihren Ärger bzw. das zurückgewiesene Kontaktbegehren gleichsam sexy (die Körperbewegungen, der Tanz), niedlich (die infantilisiert-grossen Augen, die Wasserpistole) und aggressiv (die Kampfsportelemente). Der Bitmoji-Filter macht aus einem Foto oder einem Video einen persönlichen Avatar. Das Bitmoji kann der individuellen Physiognomie angepasst werden und erscheint dann unmittelbar als eine Art Virtual Reality auf dem Display. Mit Bitmojis wird nicht nur die Körperform optimiert und vereindeutigt, sondern auch Gesten, so dass das (individualisierte> Bitmoji Stimmungen kommunizieren kann, ähnlich wie Emojis, nur eben individueller und anthropomorpher. Lara und Lara kommentieren Bitmojis im Interview, wie folgt:

Interviewauszug Lara und Lara 00.32.00-00.36.10

L1 und L2: $\quad$ Also jetzt gibt es sowas, da kannst du dich halt fotografieren und dann (machen die dich) als Bitmoji. Zum Beispiel bei mir jetzt. Bloss ich hab da ein bisschen was verändert. Zum Beispiel Pickel kann man dann halt nicht sehen. Sondern der macht dich dann halt so, wie du bist, nur halt ein bisschen schöner, weil du dann halt ein Bitmoji bist. [...] Man kann einstellen, ob man dick oder dünn ist, ob man grosse Brüste hat oder eher kleinere. Man möchte sich nicht so darstellen als ob man hässlich wäre. Also die meisten stellen sich da so dar, als wären sie super schön. 'super dünn. 

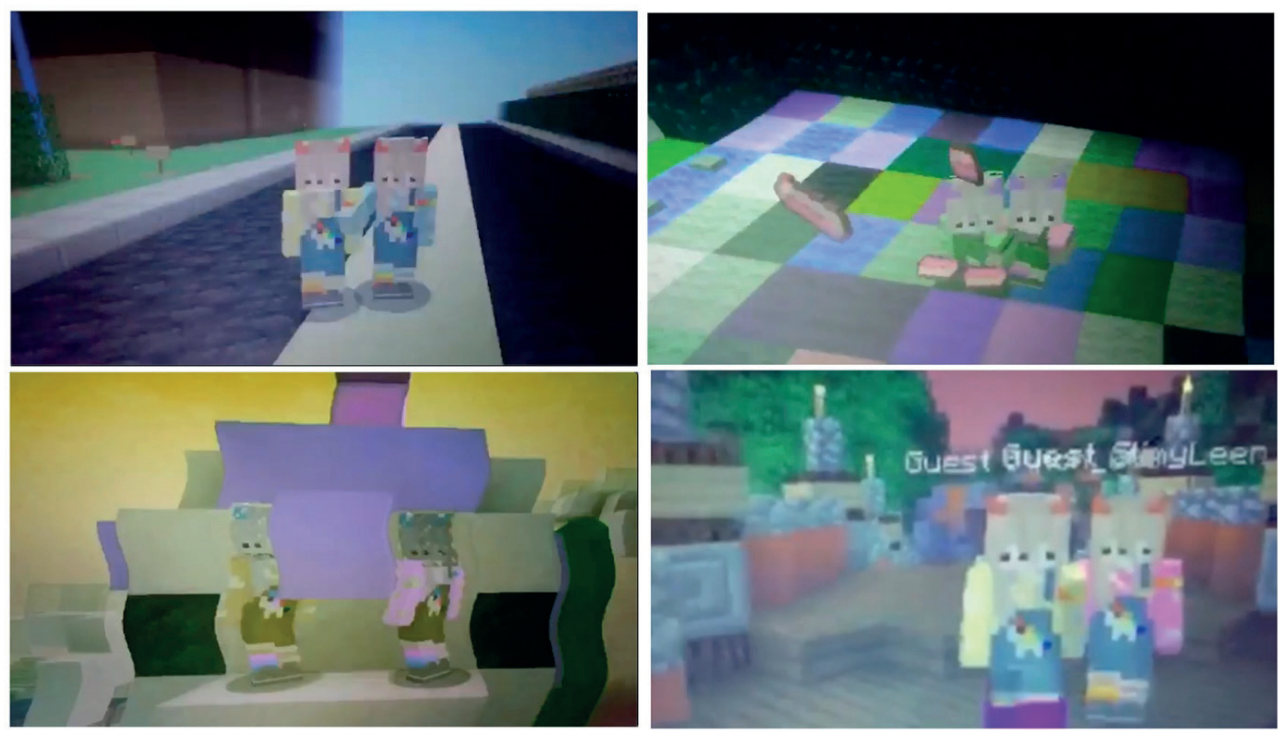

Abb. 3.: Vier Screenshots aus den Mashup-Videos (Anna Carnap u. Viktoria Flasche.

Sie grenzen sich von Mädchen ab, die sich mithilfe von Bitmojis "super schön» darstellen. Zwar wollen sie auch nicht hässlich aussehen, bspw. keine Pickel haben, aber ansonsten möglichst so aussehen, wie sie tatsächlich aussehen: «so, wie du bist». Neben diesen eher angepassten, sich einfügenden und einübenden ästhetischen Praktiken zeigen uns Lara und Lara noch ganz anders gelagerte Videos. Hierbei handelt es sich um drei einzelne Kürzestvideos, die auf der Multiplayer-Online Plattform Minecraft ${ }^{9}$ kollaborativ mit anderen inszeniert, abgefilmt, geschnitten und mit Tonspur versehen auf TikTok hochgeladen wurden (siehe Abb. 3). Im Zentrum stehen sehr ähnlich gekleidete Katzenfiguren, die in verschiedenen Settings Choreografien aufführen.

Im ersten Video wird ein Dialog inszeniert, in dem eine erwachsen klingende Person eine andere bittet, das Handy wegzulegen und etwas vom Wochenende zu erzählen. Statt zu erzählen, wird auf Snapchat verwiesen, wo «die ganzen versauten Bilder vom Wochenende» zu sehen seien. Das zweite Video beginnt mit der Frage «Did you ever look at someone and wonder: What is going on inside their head?», um dann zu drängender Elektromusik drei Katzenfiguren in verschiedenen, flackernden, schnell wechselnden Settings zu zeigen. Während die Settings in schneller, geradezu hektischer Schnittfolge wechseln, laufen die Katzen-Avatare synchron oder hüpfen im Takt gemeinsam auf und ab, während aus ihren Köpfen wie am Fliessband formlose Brocken auf die Kamera bzw. die Betrachtenden zufliegen. Im dritten Video tanzen die Kätzchen zu aggressivem Deutschrap von Schwester Ewa mit dem Text «Ihr wollt mich in Handschellen sehen».

9 Minecraft ist ein Open-World-Spiel (ursprünglich Indie-Open-World-Spiel, mittlerweile gehört es zu Microsoft). In dem Spiel können die Spieler*innen Konstruktionen aus zumeist würfelförmigen Blöcken in einer 3D-Welt bauen. 
All diesen Mashup-Videos ist gemein, den neugierigen und tendenziell übergriffigen Blick eines unkonkreten Aussenstehenden zu thematisieren und zurückzuweisen (der/diejenige, der/die einen bittet, vom Wochenende zu erzählen, oder in Handschellen sehen möchte). Die sortierte und wohl choreografierte Ordnung der Selfie-Videos wird hier sowohl formalästhetisch als auch verbal aggressiv gewendet. Verbal: Statt über die beste Freundin Auskunft zu geben (Selfie-Video 1) oder über das eigene Kontaktbegehren und den Umgang mit Verletzung/Zurückweisung/Ignoranz (Selfie-Video 2), werden hier an sie gerichtete Einsicht- und Kontrollansprüche entschieden abgelehnt. Formalästhetisch: Die Schnitte sind schnell und brüchig, die Videos flackern und wechseln abrupt in ihrer Helligkeit sowie Farbgebung. Die verwendeten Tonspuren zitieren nicht mehr etablierte Popmusik oder sanfte, wohltemperierte Stimmen, sondern abrupte und laute Samples, die die Remixlogik der Plattform in ihren Abbrüchen auch ästhetisch aufgreifen.

\subsection{Divergente Optimierungsfiguren}

Zusammengefasst bearbeiten Lara und Lara im plattformspezifischen Feld des Erscheinens zwei Optimierungsfiguren: Die Videos im Selfie-Modus folgen der formalen Logik der Porträt-Fotografie, die medienhistorisch im 19. Jahrhundert an der Herausbildung der Disziplinargesellschaft und dem bürgerlichen Subjekt mitwirkte $\left(\right.$ Schaffer 2008, 127) ${ }^{10}$.

In den Mashup-Videos zeigt sich hingegen eine Optimierungsfigur ex negativo. Diese Videos übersteigern die Logik der Plattform, die bereits auf dem Prinzip des Remix und Mashups aufbaut, also Tonspuren und Videos kreativ neu zu verbinden. In der Kombination von Genres, verschiedenen Plattformen sowie mit ihrer kollaborativen Produktionspraxis treiben Lara und Lara diese Logik weiter und wenden sie ad absurdum. Sogar im Kontext der oft hektischen, mitunter sehr (trashigen, Videoästhetik TikToks stechen diese Videos durch ihre flackernde Ästhetik hervor. Indem sie die Effekte des abgefilmten Displays in ihre Gestaltung der Videos mit aufnehmen, führen sie die Medialität des Mashups explizit auf. Auch in der Aufforderung, sich «die ganzen versauten Bilder vom Wochenende» bei Snapchat anzuschauen, oder wenn auf die neugierige Betrachtende Minecraft-Backsteine geworfen werden und (dabei) «lhr wollt mich in Handschellen sehen» gesungen wird, zeigt sich die Ablehnung eines Optimums, das an Sichtbarkeit und Berechenbarkeit geknüpft ist. Trotzdem bleiben die Videos am Optimum - wenn auch negativ - orientiert. Mit Bezug auf

10 Das auf die Individualität der/des Abgebildeten zielende Porträt war bis zur Entwicklung der Fotografie dem Adel und Klerus vorbehalten. Die «Demokratisierung des Portraits durch die Fotografie» (Schaffer 2008, 123) stellte für das aufstrebende Bürgertum ein statussicherndes Privileg dar, das Individualität und Zugehörigkeit miteinander verknüpft. Als Gegenhorizont der im Porträtfoto eingefangenen bürgerlichen Zugehörigkeit steht das typologisierende Foto der Wissenschaften, das die <rohe> Natur abbildet (und sie gleichsam bändigt): die hysterische Frau, der Südseebewohner, der Kriminelle usw. (ebd., 127). 
Richter und Allert (2017) können die Mashup-Videos als poetische Spielzüge identifiziert werden, die sich bedeutungsoffen einem rational-instrumentellen Verständnis entziehen und "problematische Situationen als Ausgangspunkt für eine Erweiterung der gemeinsamen Handlungs- und Erfahrungsspielräume nehmen» (ebd., 251). Die Erfahrung von diffus allgegenwärtigen, aber schwer zu fassenden Sichtbarkeits- und Berechenbarkeitsapellen ist die problematische Ausgangssituation, die den Spielzügen vorausgegangen ist. Spannend ist, dass die plattformlogik-konformen SelfieVideos und die, diese Logik kritisch überschreitenden Mashup-Videos an derselben Schnittstelle erzeugt wurden.

Auf Ebene der Subjektbildungsprozesse zeigen sich in diesen beiden Optimierungsfiguren - der logikkonformen und der Optimierung ex negativo - zum einen das individuierende, sich begrenzende sogenannte starke Subjekt und zum anderen das, den Widerstreit offen- und aushaltende, kollaborativ agierende hybride Subjekt (Jörissen 2017). In Abhängigkeit zu diesen Prozessen entfaltet sich Lara und Laras Freund ${ }^{\star}$ innnenschaft auf TikTok in einem divergenten Modus: Einem kreativ-kompetitivem Orientierungsmuster, das sich an die gouvernementale Ordnung der Plattform anschmiegt, steht ein kollaboratives-widerständiges Muster gegenüber, das mittels Girl-Gang-Empowerment die strukturellen Grenzen der Plattform aufgreift und ästhetisch überschreitet.

\section{4. $\quad$ Fazit}

Abschliessend können wir nun anhand dieser beiden Optimierungsfiguren, die unseren Analyseergebnissen zufolge typisch (im Sinne von eigenlogisch) für diese Schnittstelle sind, Rückschlüsse auf regimespezifische Mechanismen ziehen. Ausgehend davon, dass Regime «für ein Netz von bedeutungserzeugenden Elementen Verknüpfungsregeln bereit [stellen]» (Dimitrova et al. 2012 zitiert nach Waldmann und Aktaş 2021) und so Subjekte in ein Verhältnis zu sich selbst, zu andern und zum Dritten der Ordnung setzen (ebd.), lassen die von uns empirisch rekonstruierten Optimierungsfiguren die plattformspezifische Herrschaft der Schnittstelle konkret werden. In den hybrid hervorgebrachten Figuren des Optimums - und gerade in ihrer Negation - zeigt sich ihre machtvolle, präformierende Durchsetzungskraft. Es ist nur ein Handeln mit der Schnittstelle möglich, auch ein Handeln wider bleibt notwendig auf sie bezogen.

In Lara und Laras an der Schnittstelle hervorgebrachten Orientierungsmustern entfaltet sich ein medienspezifisches Verhältnis von Über- bzw. Unterordnung. Am Gegenhorizont der Mashup-Vidoes/Minecraft-Girls-Gang erscheinen die Nicht-Kreativen und Nicht-Unternehmungslustigen; am Gegenhorizont der Selfie-Videos die, die keine Freundin haben (bzw. niemanden anrufen können), sowie die, die «kein Glück» mit ihrer besten Freundin hatten, also die Unglücklich-Vernetzten. Denn, wer 
eine Freundin hat, die nicht witzig, sondern ernst oder humorlos ist; nicht intelligent, sondern begriffsstutzig; nicht kreativ, sondern einfallslos und nicht wunderschön, sondern unauffällig, hat Pech gehabt. Die Anderen ihrer Praxis sind diejenigen, die Pech hatten; entweder mit ihrer Freundin oder weil niemand mit ihnen befreundet sein will, eben weil sie die Anderen sind. Hier spiegelt sich die Steigerungslogik des Ranking-Algorithmus wider. Das Pech der Anderen 2.0 ist ein gesteigertes, sozusagen ein gelooptes Pech, eine geloopte Einsamkeit. Gleichzeitig scheinen die Beforschten auf die Freund*innensituation keinen Einfluss zu haben: Aus ihrer Sicht braucht es Glück, eine günstige und vernetzte Positionierung im Raum der Repräsentationen, um im steten Strom der Videos sichtbar zu werden und bleiben zu können. Oder eine Freundin mit vielen Followern. Hier wird das Regime der Freund*innenschaft zur hegemonialen Schwellenhütenden der Sichtbarkeit.

Im Sinne der Produktivität von Macht und Anerkennung (Butler 2009) dient die Freund*innenschaft, der performative Bezug auf eine gleichgeschlechtliche Gefährt*in oder auf eine Gruppe furchtloser Sichtbarkeitsrebell*innen, den beforschten 12-jährigen Mädchen, überhaupt sichtbar und insofern anerkennbar zu werden. Der Verweis auf die Freundin und der gemeinsame Auftritt lassen sie in der plattformspezifischen Sphäre des Erscheinens intelligibel und infolge handlungsmächtig werden. Die Aufführung von Zugehörigkeit ist insofern nicht nur Otheringpraktik, sie ist auch Selbstermächtigungsprogramm. Das zeigt einerseits, dass die Beforschten ein solches Programm benötigen (ihre Sichtbarkeit ist an Bedingungen geknüpft, ohne Freund ${ }^{*}$ in sind sie niemand), andererseits bietet die Freund ${ }^{*}$ in/Zugehörigkeit eine Strategie, ihrer konstitutiven Verletzlichkeit (Butler 2005, 48; Fritzsche 2015; Janssen 2018) zu begegnen. Sie sorgen durch ihre Freundin gleichsam für sich. Wenn sie ungeschützt fremdes Terrain betreten - die Erwachsenenwelt als patriarchal und heteronormativ strukturierte Öffentlichkeit - machen sie sich mit ihrer Freundin ein Stück weit unverletzlicher, unangreifbarer. Aus dieser Perspektive scheint der Verweis auf die Freundin näher am Konzept der Selbstsorge (Foucault 1989) als an der Gouvernementalität (Foucault 1977, 2000).

Neben dem Selbstsorgeaspekt tritt, durch die Produktivitätsbrille auf das Regime der Freund*innenschaft geschaut, der Ermöglichungsaspekt von Kreativität und Konnektivität hervor, und zwar gerade vor dem Hintergrund der bürgerlichen, vorstädtischen Reihenhaus-Realität, von der uns die Beforschten im weiteren Interview berichten. Der prinzipiell globalvernetzte Möglichkeitsraum der Social Media Plattform mit ihren Aktivierungs-, Affizierungs- und Involvierungsappellen (siehe auch den TikTok Slogan: «Make Your Day»), bietet Lara und Lara eine geschützte Option der Alteritätserfahrung, d. h. der Möglichkeit, sich von der Welt - in kurzweiligen Kürzestvideos und medialer Distanz - irritieren zu lassen und mit den Mashup-Videos wiederum andere zu irritieren. Kulturaffirmativ zugespitzt formuliert ist es besser (im 
Sinne von bildsamer), sich auf dem Minecraft-Server zu treffen, einen Musikvideoclip zu choreografieren, zu schneiden und zu verschlagworten, als sich untätig und vereinzelt dem Nachmittagsprogramm im Fernsehen zu überlassen.

Auch in der theoretischen Reflexion bleiben das starke und hybride Subjekt (Jörissen 2017) uneindeutig, tendenziell bezugslos nebeneinander stehen und zeigen, «dass der freiwillige Zwang zur Optimierung durch kreative Selbstbewirtschaftung und die Effizienzsteigerung von Feedbackschleifen nicht im Widerspruch» (Waldmann und Aktaş 2021) zueinander stehen; sie kulminieren und pluralisieren gleichsam in der notwendigen wie emanzipativen Sichtbarkeit an der Schnittstelle.

\section{Literatur}

Albert, Matthias, Klaus Hurrelmann, und Gudrun Quenzel. 2019. 18. Shell Jugendstudie. Eine Generation meldet sich zu Wort. Weinheim u. Basel: Beltz Juventa.

Berry, David. M. 2014. Critical Theory and the Digital. New York: Bloomsbury.

Bloch, Ernst. 1963. Tübinger Einleitung in die Philosophie. Frankfurt/M.: Suhrkamp.

Boyd, Danah. 2014. It's Complicated. The social Lives of networked teens. New Haven u. London: Yale University Press.

Breljak, Anja, und Rainer Mühlhoff. 2019. «Was ist Sozialtheorie der Digitalen Gesellschaft?» In Affekt Macht Netz, herausgegeben von Rainer Mühlhoff, Anja Breljak, und Jan Slaby, 7-34. Bielefeld: transcript Verlag. https://doi.org/10.14361/9783839444399-001.

Bröckling, Ulrich. 2007. Das unternehmerische Selbst: Soziologie einer Subjektivierungsform. Frankfurt/M.: Suhrkamp.

Bublitz, Hannelore. 2010. Im Beichtstuhl der Medien. Bielefeld: Transcript. https://doi. org/10.14361/transcript.9783839413715.

Butler, Judith. 2005. Gefährdetes Leben. Politische Essays. Frankfurt a. M.: Suhrkamp

Butler, Judith. 2009. Die Macht der Geschlechternormen und die Grenzen des Menschlichen. Frankfurt a. M.: Suhrkamp

Butler, Judith. 2010. Raster des Krieges. Warum wir nicht jedes Leid beklagen. Frankfurt/M. u. New York: Campus Verlag.

Carnap, Anna, und Viktoria Flasche. 2020. «Diskursive Sichtbarkeiten - Aufführungen von Geschlechtlichkeit in (post)digitalen Jugendkulturen» In Geschlecht und Medien. Räume, Deutungen, Repräsentationen, herausgegeben von Britta Hoffahrt, Eva Reuter und Susanne Richter, Frankfurt/M. u. New York: Campus Verlag.

Castells, Manuel. 2008. Communication Power. Oxford: Oxford University Press.

Cramer, Florian. 2014. «What Is 'Post-Digital'?» A Peer-Reviewed Journal About 3 (1): 10-24. https://doi.org/10.7146/aprja.v3i1.116068.

Dimitrova, Petja, Eva Egermann, Tom Holert, Jens Kastner, und Johanna Schaffner. 2012. Regime. Wie Dominanz organisiert und Ausdruck formalisiert wird. Münster: Ed. Assemblage. 
Engel, Juliane, und Benjamin Jörissen. 2019. «Unsichtbare Sichtbarkeiten: Kontrollverlust und Kontrollphantasmen in öffentlichen und jugendkulturellen Digitalisierungsdiagnosen». In Gegenwartsdiagnosen, herausgegeben von Thomas Alkemeyer, Nikolaus Buschmann, und Thomas Etzemüller, 549-68. Bielefeld: transcript Verlag. https://doi. org/10.14361/9783839441343-028.

Feierabend, Sabine, Thomas Rathgeb, Hediye Kheredmand, und Stephan Glöckler. 2020. «JIMStudie 2019. Jugend, Information, Medien. Basisuntersuchung zum Medienumgang 12bis 19-Jähriger». Herausgegeben von Medienpädagogischer Forschungsverbund Südwest (mpfs). Medienpädagogischer Forschungsverbund Südwest (LFK, LMK). https://www.mpfs. de/fileadmin/files/Studien/JIM/2019/JIM_2019.pdf.

Firsching, Jan. 2020. TikTok Statistiken 2020: 100 Mio. Nutzer in Europa \& über 800 Mio. weltweit. Zugriff 16.02.2021. https://www.futurebiz.de/artikel/tiktok-statistiken-2019/.

Forster, Edgar. 2005. «Männerforschung, Gender Studies und Patriarchatskritik.»In Geschlechterforschung in der Kritik. herausgegeben von Rita Casale, Barbara Rendtorff, Sabine Andresen, Eva Moser und Annedore Prengel. Jahrbuch Frauen- und Geschlechterforschung in der Erziehungswissenschaft, 2005/1, 41-72. Opladen u. Bloomfield Hills: Budrich.

Foucault, Michel. 1977. Überwachen und Strafen. Die Geburt des Gefängnisses. Frankfurt/M.: Suhrkamp.

Foucault, Michel. 1989. Die Sorge um sich. Sexualität und Wahrheit. Frankfurt/M.: Suhrkamp.

Foucault, Michel. 2000. «Die Gouvernementalität.»In Gouvernementalität der Gegenwart. Studien zur Ökonomisierung des Sozialen, herausgegeben von Thomas Lemke, Ulrich Bröckling und Susanne Krasmann, 41-67. Frankfurt/M.: Suhrkamp.

Fritzsche, Bettina. 2016. «Die Relevanz der Kategorie Geschlecht bei schulischen Regulationen von Verletzbarkeit». Feministische Studien 29(2), 280-292. https://doi.org/10.1515/fs-20110210.

Hall, Stuart. 1979. »Culture, the Media and the ,Ideological Effect'«In Mass Communication and Society, herausgegeben von James Curran, Michael Gurevitch u. Janet Wollacott, 315-348. London: Hodder Arnolds.

Hall, Stuart. 1989. "Reflektionen über das Kodieren/Dekodieren-Modell. Ein Interview». In Ideologie, Identität, Repräsentation: Ausgewählte Schriften, herausgegeben von Stuart Hall, Bd. 4, 81-136. Berlin: Argument Verlag.

Hepp, Andreas, und Uwe Hasebrink. 2014. «Human interaction and communicative figurations. The transformation of mediatized cultures and societies». In Mediatization of Communication. herausgegeben von Knut Lundby, 249-272. Berlin, New York: De Gruyter. https://doi. org/10.1515/9783110272215.249.

Hoffarth, Britta. 2012. «Dispositiv 2.0: Wie Subjekte sich im Web 2.0 selbst und gegenseitig regieren.» In Mediendiskursanalyse, herausgegeben von Philipp Dreesen, Łukasz Kumięga und Constanze Spieß, 207-227. Wiesbaden: VS Verlag. https://doi.org/10.1007/978-3-53193148-7_9.

Janssen, Angela. 2018. Verletzbare Subjekte. Leverkusen: Budrich. 
Jörissen, Benjamin. 2011. «Medienbildung - Begriffsverständnisse und -reichweiten». In Medienbildung und Medienkompetenz, herausgegeben von Heinz Moser, Petra Grell und Horst Niesyto. München: Kopaed. https://doi.org/10.21240/mpaed/20/2011.09.20.X.

Jörissen, Benjamin. 2014. «Bildung der Dinge: Design und Subjektivation». In Subjekt Medium Bildung, herausgegeben von Benjamin Jörissen und Thosten. Meyer, 215-234. Wiesbaden: VS Verlag. https://doi.org/10.1007/978-3-658-06171-5_11.

Jörissen, Benjamin. 2016. «Digitale Bildung〉 Und Die Genealogie Digitaler Kultur: Historiographische Skizzen». MedienPädagogik: Zeitschrift für Theorie Und Praxis Der Medienbildung 25 (Computer Science Education):26-40. https://doi.org/10.21240/mpaed/25/2016.10.26.X.

Jörissen, Benjamin 2017. «Subjektivation und ,ästhetische Freiheit' in der post-digitalen Kultur». In Das starke Subjekt herausgegeben von Tom Braun, Max Fuchs und Gerd Taube, 187-200. München: Kopaed.

Jörissen, Benjamin. 2018. "Subjektivation und ästhetische Bildung in der post-digitalen Kultur». Vierteljahresschrift für wissenschaftliche Pädagogik. 2018(94): 51-70. https://doi. org/10.30965/25890581-0940100.

Jörissen, Benjamin. 2020. «Ästhetische Bildung im Regime des Komputablen». Zeitschrift für Pädagogik 66(29): 341-355. https://doi.org/10.3262/ZP2003341.

Jörissen, Benjamin, Karoline Schröder, und Anna Carnap. 2020. «Postdigitale Jugendkultur: Kernergebnisse einer qualitativen Studie zu Transformationen ästhetischer und künstlerischer Praktiken». In Kulturelle Bildung: Theoretische Perspektiven, methodologische Herausforderungen, empirische Befunde, herausgegeben von Susanne Timm, Jana Costa, Claudia Kühn und Annette Scheunpflug, 61-78. Münster u. New York: Waxmann.

LeCun, Yann, Joshua Bengio, und Goeffrey Hinton. 2015. «Deep Learning». Nature 521. 28. Mai 2015, S. 436-444. https://doi.org/10.1038/nature14539.

Latour, Bruno. 1998. «Über technische Vermittlung.» In Technik und Sozialtheorie herausgegeben von Werner Rammert, 29-81. Frankfurt/M: Campus.

Lury, Celia. 1997. Prosthetic Culture. Photography, Memory, Identity. London: Routledge.

Marchart, Oliver. 2003. «Warum Cultural Studies vieles sind, aber nicht alles. Zum Kultur- und Medienbegriff der Cultural Studies». Medienheft, Dossier 19, 7-14. https://www.medienheft.ch/dossier/bibliothek/d19_MarchartOliver.pdf.

McRobbie, Angela. 2010. Top Girls: Feminismus und der Aufstieg des neoliberalen Geschlechterregimes. Wiesbaden: VS Verlag.

Medina Serrano, Juan C., Orestis Papakyriakopoulos, und Simon Hegelich. 2020. «Dancing to the Partisan Beat: A First Analysis of Political Communication on TikTok». Web Science. https://doi.org/10.1145/3394231.3397916.

Meyer, Thorsten. 2012. «Das Weltweit-Werden und der umgestülpte Cyberspace», In geo@web: Medium, Räumlichkeiten und geographische Bildung, herausgegeben von Inga Gryl, Tobias Nehrdich und Robert Vogler, 201-214. Wiesbaden: VS Verlag. https://doi.org/10.1007/9783-531-18699-3_11.

Nassehi, Armin. 2019. Muster. Theorie der digitalen Gesellschaft. München: C.H. Beck. 
Nohl, Arnd-Michael. 2012. «Be-Dingte Bildung?» In Qualitative Bildungsforschung und Bildungstheorie herausgegeben von Ingrid Miethe und Hans-Rüdiger Müller, 227-245. Opladen u. Bloomfield Hills: Budrich.

Nohl, Arnd-Michael. 2011. Pädagogik der Dinge. Bad Heilbrunn: Klinkhardt.

Nohl, Arnd-Michael. 2017. «Die empirische Rekonstruktion materieller Artefakte mit der Dokumentarischen Methode». In Dinge und Raum in der qualitativen Bildungs- und Biographieforschung herausgegeben von Anja Tervooren und Robert Kreitz, 37-54. Opladen u. Bloomfield Hills: Budrich.

Poell, Thomas. 2020. «Three Challenges for Media Studies in the Age of Plattform». Television \& New Media 2(6), 650-657. https://doi.org/10.1177\%2F1527476420918833.

Porombka, Stephan. 2016. "Sekundentricks». In where the magic happens. Bildung nach der Entgrenzung der Künste herausgegeben von Torsten Meyer, Julia Dick, Peter Moormann und Julia Ziegenbein. 27-35. München: Kopaed.

Poster, Mark. 2008. «Die Sorge um sich im Hyperrealen». Paragrana, 17(2008), 201-227. https://doi.org/10.1524/para.2008.0012.

Przyborski, Aglaja. 2018. Bildkommunikation. Qualitative Bild- und Medienforschung. Berlin u. Boston: De Gruyter. https://doi.org/10.1515/9783110501704.

Reckwitz, Andreas. 2008. Subjekt. Bielefeld: Transcript. https://doi. org/10.14361/9783839405703.

Reuter, Markus, und Chris Koever. 2019. «Gute Laune und Zensur». Zugriff 29.09.2020. https:// netzpolitik.org/2019/gute-laune-und-zensur/.

Richter, Christoph, und Heidrun Allert. 2017. «Poetische Spielzüge als Bildungsoption in einer Kultur der Digitalität». In Digitalität und Selbst, herausgegeben von Heidrun Allert, Michael Asmussen und Christoph Richter. 237-262. Bielefeld: Transcript. https://doi. org/10.14361/9783839439456-011.

Roberge, Jonathan, und Robert Seyfert. 2017. «Was sind Algorithmuskulturen?» In Algorithmuskulturen. Über die rechnerische Konstruktion der Wirklichkeit, herausgegeben von Jonathan Roberge und Robert Seyfert, 7-40. Bielefeld: Transcript. https://doi. org/10.14361/9783839438008-001.

Schade, Sigrid, und Silke Wenk. 2011. Studien zur visuellen Kultur. Einführung in ein transdisziplinäres Forschungsfeld. Bielefeld: Transcript. https://doi.org/10.14361/ transcript.9783839409909.

Schaffer, Johanna. 2008. Ambivalenzen der Sichtbarkeit. Über die visuellen Strukturen der Anerkennung. Bielefeld: Transcript. https://doi.org/10.14361/9783839409930.

Schreiber, Maria. 2020. Digitale Bildpraktiken. Handlungsdimensionen visueller vernetzter Kommunikation. Wiesbaden: VS Verlag. https://doi.org/10.1007/978-3-658-30788-2.

Sekula, Allan 2003. «Der Körper und das Archiv». In Diskurse der Fotografie, herausgegeben von Herta Wolf. Bd. 2. 269-334. Frankfurt/M.: Suhrkamp.

Silverman, Kaja. 1997. «Dem Blickregime begegnen». In Privileg Blick, herausgegeben von Christian Krawagna, 41-64. Berlin: Ed. ID-Archiv. 
Stalder, Felix. 2016. Kultur der Digitalität, Berlin: Suhrkamp.

Stiegler, Bernard. 2009. Technik und Zeit. Band 1. Der Fehler des Epimetheus. Berlin: Diaphanes.

TikTok. 2021. «TikTok 〈For Business`». Zugriff 16.02.2021. https://www.tiktokforbusinesseurope.com/de/measurement.

Traue, Boris. 2013. «Visuelle Diskursanalyse. Ein pragmatischer Vorschlag zur Untersuchung von Sicht- und Sagbarkeiten im Medienwandel». ZfD - Zeitschrift für Diskursforschung, 2,1 (2013), 117-136. Weinheim: Beltz.

VanDijck, José, und Thomas Poell. 2013. «Understanding Social Media Logic». In Media and Communication. 1(1), 2-14. https://doi.org/10.12924/mac2013.01010002.

Villi, Mikko. 2013. «Publishing and Messaging Camera Phone Photographs: Patterns of Visual Mobile Communication on the Internet». In Mobile Media Practices, Presence and Politics: The Challenge of Being Seamlessly Mobile, herausgegeben von Kathleen Cumiskey und Larissa Hjorth, 214-228. New York, London: Routledge.

Waldmann, Maximilian, und Ulaş Aktaş. 2021. «Hegemonialität und Gouvernementalität von Optimierungsverhältnissen. Prekäre postdigitale Subjektivierungspraktiken zwischen Affirmation und Widerständigkeit». MedienPädagogik: Zeitschrift für Theorie Und Praxis Der Medienbildung 42 (Optimierung):327-48. https://doi.org/10.21240/mpaed/42/2021.08.27.X. 